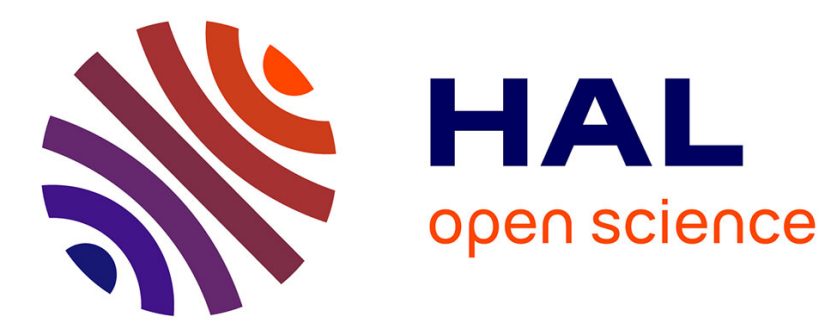

\title{
De l'usage du risque dans le gouvernement du crime
} Jean-François Cauchie, Gilles Chantraine

\section{To cite this version:}

Jean-François Cauchie, Gilles Chantraine. De l'usage du risque dans le gouvernement du crime. Champ Pénal, 2005, Champ pénal, 2, 10.4000/champpenal.80 . hal-02441940v2

\section{HAL Id: hal-02441940 \\ https://hal.univ-lille.fr/hal-02441940v2}

Submitted on 14 May 2021

HAL is a multi-disciplinary open access archive for the deposit and dissemination of scientific research documents, whether they are published or not. The documents may come from teaching and research institutions in France or abroad, or from public or private research centers.
L'archive ouverte pluridisciplinaire HAL, est destinée au dépôt et à la diffusion de documents scientifiques de niveau recherche, publiés ou non, émanant des établissements d'enseignement et de recherche français ou étrangers, des laboratoires publics ou privés. 


\section{De l'usage du risque dans le gouvernement du}

\section{crime}

Nouveau prudentialisme et nouvelle pénologie

Jean-François Cauchie et Gilles Chantraine

\section{OpenEdition}

\section{Journals}

\section{Édition électronique}

URL : http://journals.openedition.org/champpenal/80

DOI : 10.4000/champpenal.80

ISBN : 978-2-8218-0586-6

ISSN : $1777-5272$

\section{Éditeur}

Association Champ pénal / Penal field

Ce document vous est offert par Université de Lille

\section{Université de Lille}

\section{Référence électronique}

Jean-François Cauchie et Gilles Chantraine, "De l'usage du risque dans le gouvernement du crime », Champ pénal/Penal field [En ligne], Vol. II | 2005, mis en ligne le 13 mai 2005, consulté le 10 janvier 2020. URL : http://journals.openedition.org/champpenal/80 ; DOI : 10.4000/champpenal.80

Ce document a été généré automatiquement le 10 janvier 2020.

(c) Champ pénal 


\title{
De l'usage du risque dans le gouvernement du crime
}

\author{
Nouveau prudentialisme et nouvelle pénologie
}

Jean-François Cauchie et Gilles Chantraine

Nous remercions l'évaluateur anonyme de Champ Pénal/Penal Field, notre collègue Dan Kaminski ainsi que les participants au colloque " Acteur, risque et prise de risque » organisé par le Clersé à l'Université de Lille 1 les 25 et 26 novembre 2004. Si leurs remarques ont contribué à enrichir cet article, elles ne nous dédouanent évidemment en rien de l'entière responsabilité de nos propos.

1 La perspective dite " gouvernementaliste ${ }^{1}$ » offre un cadre d'analyse fécond au chercheur qui, s'intéressant aux politiques publiques contemporaines en matière de délinquance, est tôt ou tard confronté, de gré ou de force pourrait-on dire, à la notion de «risque ». En effet, cette perspective permet de prendre acte, entre autres choses, de la grande variété des domaines où l'outil risque est mobilisé dans le champ du contrôle du crime (prévention, prédiction, traitement du crime, etc.), ainsi que de la grande polymorphie normative qui peut être faite de l'usage du risque ${ }^{2}$ : les politiques de "prévention situationnelle » des risques ne sont pas l'équivalent d'une politique de réduction des risques en matière de drogues, tout comme la " gestion actuarielle des risques » n'est pas l'équivalent d'un traitement dynamique et personnalisé des « risques » que présente une personne incarcérée. Par là, cette perspective générale s'oppose formellement aux théories de la «société du risque » qui posent le risque comme le point d'orgue d'une analyse macrosociologique trop uniformisante (Beck, 2001). La notion de risque, dans une perspective gouvernementaliste, est davantage une porte d'entrée qu'un point d'orgue (ou pire un paradigme), porte d'entrée qui débouche sur le corridor analytique de « régimes de gouvernement » particuliers. Elle postule que bien qu'il soit le socle d'une forme singulière de savoir, un "risque " n'a pas de signification en soi : il ne prend réellement sens qu'en s'insérant dans des logiques d'action et des rationalités spécifiques qui elles constituent le véritable horizon de l'analyse.

2 Au sens où nous l'entendrons ici, la gouvernementalité est coextensive au champ sémantique de la notion de gouvernement (Senellart, 2004, 406-407), entendue au sens 
large de techniques et procédures destinées à diriger la conduite des hommes (Foucault, 1980). Elle est une forme d'activité ayant pour objectif d'influencer, de guider ou d'affecter les conduites d'une personne ou de personnes (Gordon, 1991). Le projet général de recherches sur la gouvernementalité consiste dans ce cadre à explorer les différentes pratiques par lesquelles les autorités et divers organismes sociaux, communautaires et politiques tentent de diriger les actions des individus et des populations au nom d'idées éthiques, de fins politiques, d'une nécessité économique ou de buts sociaux (Dean, 1999, 132-133). Ce projet général sous-entend, dans la continuité critique des travaux de M. Foucault, que les régimes de gouvernement peuvent évidemment être incarnés dans des dispositifs étatiques mais également dans les pratiques de toute une série d'autres acteurs: familles, agences privées, mouvements sociaux, collectifs divers, systèmes sociaux. Le "succès " d'un régime de gouvernement dépend ainsi d'une variété d'alliances et de compromis entre divers corps d'expertises, entre divers critères de jugement et enjeux techniques (Rose, 2000, 323). Dans ce cadre, les adeptes de la gouvernementalité proposent des formes d'articulations originales entre, d'un côté, des stratégies gouvernementales - le gouvernement des autres - et, d'un autre côté, les formes de subjectivations individuelles - le gouvernement de soi. Il s'agit alors d'identifier des régimes de gouvernement en révélant la manière par laquelle leurs modes d'exercice du pouvoir dépendent de manières spécifiques de penser et d'agir, de gouverner des populations, mais également d'objectiver des individus et de produire de la subjectivité.

On l'a annoncé, les usages sociaux du risque sont, en matière pénale, hétérogènes, et notre objectif n'est pas d'en dresser un inventaire exhaustif. Nous ciblerons plutôt notre présentation en mettant en perspective deux régimes de gouvernement spécifiques, conceptualisés sous le vocable du nouveau prudentialisme (O'Malley, 1992) et de la nouvelle pénologie (Feeley, Simon, 1992) ; le premier se situe au niveau de la prévention du crime, le second au niveau de son traitement. Nous les mettrons en débat en les présentant sous des formes idéale-typiques, illustrées néanmoins par quelques exemples concrets. Cette présentation idéale-typique ne doit pas - insistons encore - conduire le lecteur à sousestimer les tensions et les fractures idéologiques à l'intérieur même du champ pénal, ainsi que la coexistence simultanée, dans les discours comme dans les pratiques, de divers régimes de gouvernement ${ }^{3}$. L'intérêt de la démarche idéale-typique, visant à décrire les traits marquants de ces stratégies en émergence, est plutôt d'outiller les réflexions et les observations empiriques ultérieures dans des contextes nationaux et socioculturels différenciés. Sans détailler ici ces réalités nationales hétérogènes, soulignons simplement ici que si le nouveau prudentialisme et la nouvelle pénologie sont surtout palpables dans les pays anglo-saxons comme les USA, l'Australie, le Canada, l'Angleterre (Feeley, Simon, 1992 ; O’Malley, 1992 ; Crawford, 2001), des études en constatent néanmoins la présence, certes timide mais néanmoins palpable et grandissante, dans des pays comme la France ou la Belgique (Brion, 2001, 2003 ; Mary, 2001 ; Kaminski, 2002 ; Chantraine, 2004b).

4 Au-delà de la nécessité d'analyser une catégorie qui, de fait, est de plus en plus mobilisée par les acteurs, notre curiosité théorique pour ce processus a largement été attisée par le fait que là où le gouvernement du crime est souvent présenté comme ayant une forte dimension morale, expressive et émotive, la catégorie du risque est au contraire, elle, fréquemment assimilée à un pur outil technique. $\mathrm{Y}$ a-t-il dès lors paradoxe entre cette action gouvernementale et son outil ? Doit-on plutôt comprendre que l'usage du risque dans le gouvernement du crime renvoie à la technicisation de ce dernier ? La légitimité 
du système pénal passerait-elle désormais par un assoupissement d'objectifs aussi valeureux qu'aléatoires (comme l'aide aux infracteurs), assoupissement qui serait couplé au réveil d'objectifs strictement endogènes comme la productivité interne, l'efficience et le consumérisme (Kaminski, 2002) ? Faut-il au contraire supposer que l'usage du risque maintient voire renforce la dimension morale / moralisante des régimes qui gouvernent le crime, et si oui comment? (Hunt, 2003, 165).

Dans le cadre des deux régimes de gouvernement étudiés, nous montrerons ainsi que l'usage du risque revêt ce que l'on appellera une dimension "biface ", dimension qui suggère l'existence d'un processus de reconfiguration et de déplacement de la question morale autour d'acteurs, de pratiques, de problèmes et de contradictions en émergence au cœur du gouvernement du crime. D'un côté, en effet, l'usage du risque semble déconnecter la prévention et le traitement du crime de questions " morales » au sens où la délinquance et sa gestion ne sont plus saisies comme des problèmes "sociaux ", mais plutôt comme de simples problème d'efficacité technocratique et statistique. De l'autre, on observe un processus de (re)moralisation distillée à travers une responsabilisation accrue à la fois des délinquants et des victimes potentielles. Suite à l'identification de cette dimension biface, on explorera alors l'hypothèse selon laquelle le nouveau prudentialisme et la nouvelle pénologie traduisent bien plus qu'une simple technicisation du gouvernement du crime, et ce contrairement à ce que certaines conceptualisations originelles le laissent parfois supposer. En bref, on observerait le processus dual suivant : d'une part, une éthique de la modération qui glisse vers une efficacité procédurale et organisationnelle de la répression (une efficacité qui se présente comme neutre et dépouillée de toute valeur morale); de l'autre, une coproduction de normes et de valeurs partagées sur un espace public qui glisse vers un retrait prudent et moralisateur dans la sphère privée. En préalable à l'exploration de ce double processus, il convient de le relativiser en rappelant que bien qu'elle apparaisse aujourd'hui sous une forme inédite, la concurrence des dimensions morales, émotives et moralisantes par des dimensions plus techniques et managériales n'a pas attendu l'émergence de l'outil « risque » pour exister et modeler le gouvernement du crime.

\section{Gouvernement du crime entre punition morale et contrôle technique}

6 Durkheim, on le sait, a défendu la thèse selon laquelle les sociétés modernes, comme celles qui les ont précédées, voient dans les notions de crime et de peine une contribution à la vitalité d'une conscience collective ainsi qu'une assise au maintien et au rappel d'un ordre moral dominant. Expiatoire et finalement surtout destiné aux « honnêtes gens ", le châtiment viserait donc moins à rééduquer des individus antisociaux ou à intimider des imitateurs possibles qu'à retrouver une solidarité perdue.

\section{Qui a parlé d'une conscience et d'une solidarité collectives ?}

7 Il nous paraît important de nuancer cette thèse durkheimienne. Dans une critique puissante de la justice punitive et une réfutation de son utilité sociale, Mead (1918) a montré que la solidarité dont parle Durkheim $(1986,1992)$ est d'abord une solidarité émotionnelle et agressive qui renvoie à la production d'une moralité guerrière peu propice à restaurer une cohésion sociale à laquelle appartiendrait l'infracteur. De plus, les 
notions durkheimiennes de conscience collective et d'ordre moral prêtent aisément à la critique, et ceci plus encore dans nos sociétés hautement complexes et différenciées. $\mathrm{Si}$ Durkheim a pu reconnaître que les sentiments collectifs pouvaient évoluer dans le temps et l'espace (pour l'auteur, un acte ne froisse pas la conscience commune parce qu'il est un crime, il est un crime parce qu'il froisse la conscience commune) et s'il n'a jamais suggéré explicitement que les sociétés modernes présentaient un consensus total, il n'en a pas moins laissé entendre qu'un État incapable de porter les sentiments collectifs de toute une société serait pathologique et qu'il ne pourrait se maintenir. Or, comme l'a montré D. Garland, le monde actuel suggère une interprétation différente : « la présence des conflits de groupe à long terme - basés sur la classe, la race, le sexe, l'identité régionale et l'idéologie - est une qualité inhérente de la plupart des sociétés modernes et ces dernières restent pourtant capables de fonctionner, de persister à travers le temps et de se reproduire. Une société peut donc se maintenir sans avoir une conception universelle de l'ordre moral » (Garland, 1990, 50-51, notre traduction). En déconstruisant la notion de déviance (et en lui retirant ainsi son caractère intrinsèque, naturel), H. Becker rappelle, dans le même sens, qu'il n'y a pas (ou très peu) de normes communément admises par tous les membres d'une société. Ce qui est interdit dans un groupe social peut en effet être hautement valorisé dans un autre. Cela ne veut pas dire qu'il n'y ait pas de modèle général d'imposition culturelle uniforme à une société. Mais plutôt que de parler d'une conscience collective qui réunirait, tout en les transcendant, les divers groupes sociaux, il serait plus juste d'affirmer "que l'idéologie dominante n'opère que comme mécanisme culturel de fédération et de cohésion des groupes dominants, plutôt que comme un mécanisme de fabrication de la conscience des dominés" (Martucelli, 2004, 471). Il faudrait donc reconnaître "qu'il n'y a pas nécessairement de 'naturalisation de la domination', ni d'adhésion 'spirituelle' des dominés, mais tout simplement un accord, au moins apparent, entre leurs pratiques et l'ordre social en place ; une acceptation pratique qui n'empêcherait pas les contestations plus ou moins cachées de s'exprimer " (Martucelli, 2004, 473).

\section{D'une rhétorique de la morale à une rhétorique de l'efficace ?}

8 Faut-il pour autant voir dans la société moderne une société « sans morale » et dépourvue de sentiments collectifs ? Certainement pas, mais alors qu'Elias (1975) évoque une société moderne qui se gouverne à travers une force morale, et plus précisément à travers un système d'institutions qui coopèrent et se complètent pour imposer un ordre normatif plus ou moins partagé, Bauman $(2002,62)$ va y ajouter une autre qualité : à savoir, la tendance moderne à refouler, censurer et délégitimer les motivations morales et éthiques de l'action sociale, et ce au nom d'une rationalité parfois dévastatrice. Pour lui, le processus moderne de civilisation consisterait entre autres à dépouiller l'utilisation de la violence de toute valeur morale et à débarrasser tout désir de rationalité de toute interférence de normes éthiques ou d'inhibitions d'ordre moral (Bauman, 2002, 62). La thèse de Bauman pourrait expliquer que les régimes de gouvernement glissent progressivement d'une rhétorique de la morale à une rhétorique de l'efficace, glissement au cours duquel les valeurs morales (et immorales) sont reléguées à l'extérieur d'actions conçues comme rationnelles et purement rationnelles. Désenchantée, l'action sociale ne renverrait plus à des décisions bonnes ou mauvaises, justes ou injustes, mais uniquement à des actions mesurables à l'aune de valeurs techniques et procédurales. 
Face à la thèse baumanienne (héritée entre autres de l'Ecole de Francfort et autre Weber), il ne faudrait cependant pas sous-estimer la force des analyses durkheimiennes. Durkheim nous permet en effet de voir que dans les régimes de gouvernement du crime, réside autre chose qu'un simple business du contrôle du crime, interprétation réductionniste en vogue, notamment à travers la notion $\mathrm{d}^{\text {' } ~}$ industrie carcérale $»^{4}$. Les dimensions morale, émotive et moralisante dont restent assurément empreints les régimes de gouvernement du crime semblent néanmoins avoir leurs espaces privilégiés. Alors que la justice se veut expressive dans les Cours, les tribunaux et les médias, elle paraît toujours plus technicisée dans la prévention, le traitement et la prédiction du crime. La Cour devenant le forum où « justice est faite ", les dispositifs en aval (prisons, services de probation, services de libération conditionnelle) seraient désormais perçus et s'autodéfiniraient comme appareils techniques, plus concernés par le management administratif que par la gouvernance éthico-politique et les rituels publics (Garland, 1990, 71-72).

10 Ce dernier aspect est palpable par exemple dans la manière où, confronté aux critiques de son environnement, le système pénal a réagi en redéfinissant ses buts ainsi que ses notions de "succès " et d' "échec " (Kaminski, 2002). C'est ainsi que l'administration pénitentiaire ne fait plus toujours l'effort de s'engager dans la réhabilitation ou, si elle entretient cet espoir, veille en tout cas à ne pas en faire un indicateur de performance (Chantraine, 2004a). Dans un tel cas de figure, ce ne sont pas la réhabilitation et la revalorisation du justiciable qui soutiennent l'idéologie générale du système pénal (Garland, 1998). Les critères de légitimation du système seront de plus en plus sélectionnés en vertu de leur capacité à évaluer des performances " évaluables " par le système (Dupont, Ratcliffe, 2000, 229). En ce sens, les évaluations porteront davantage sur l'effectivité des procédures mises en place que sur les résultats qu'elles peuvent produire (Dean, 1999). Comme l'explique Garland $(1998,60)$ :

«Les nouveaux indicateurs de performance mesurent ce que l'organisation 'fait' plutôt que, faute de mieux, ce qu'elle 'réussit'. Ainsi, les nouveaux objectifs de la direction en matière de rationalisation, de rentabilité et de relations aux clients en viennent peu à peu à remplacer l'objectif social de la réduction de la criminalité, qui était le but initial du système et de son pouvoir. Le système échouant dans les buts qu'il s'était donnés, celui-ci, dans une sorte de défense organisationnelle bureaucratique, change ses objectifs et s'en donne de nouveaux qui lui conviennent mieux et qu'il peut atteindre.»

11 Pouvons-nous pour autant diagnostiquer ou prophétiser, de manière radicale, la présence accrue de régimes de gouvernement du crime qui se positionne(ro)nt en dehors de toute interrogation morale ? Pouvons-nous parler des tribunaux comme du dernier bastion moral de ce type de gouvernement et nous risquer à évoquer l'actuelle (ou la prochaine) transition de la punition morale vers le contrôle technique? Les points suivants vont apporter une réponse négative à ces questions.

\section{Risque et Gouvernement du crime}

12 Après avoir défini le nouveau prudentialisme (O'Malley, 1992) et la nouvelle pénologie (Feeley, Simon, 1992) sous une forme idéal-typique, nous montrerons que les territoires respectifs des dimensions morales et techniques sont moins étanches qu'il n'y parait. 


\section{Deux idéaux-types : nouveau prudentialisme et nouvelle pénologie}

13 Davantage situé au niveau de la prévention du crime, le nouveau prudentialisme, conceptualisé par O'Malley (1992), renvoie d'abord à une construction de gouvernement qui supprime la conception clé de réguler des individus par la gestion de risques collectifs et la remplace en assignant aux individus isolés la responsabilité de la gestion de leurs risques. Cette assignation peut cependant échouer pour des raisons de volonté ou d'incapacité. La prudence recommandera alors une «neutralisation » des récalcitrants et autres «incapables». Mais le concept de nouveau prudentialisme renvoie aussi à la sécurisation des situations ou lieux identifiés comme " criminogènes ", au sens où ils font l'objet d'un calcul de risques énonçant une (haute) probabilité qu'ils soient le théâtre de crimes : parkings non surveillés, parcs, carrefours déserts, autoroutes, quartiers isolés, stades de football, arrêts de bus, etc.

14 Sur quelle base ce régime de gouvernement diffère-t-il de l'ancien prudentialisme ? Sur la même base que celle qui distingue le libéralisme du néolibéralisme (Wendy Brown, 2004) ${ }^{5}$ : au contraire d'un libéralisme classique qui maintenait une distinction, et parfois même une tension, entre les critères de la morale individuelle ou collective et les actions économiques, le néolibéralisme façonnerait normativement les individus comme des acteurs entrepreneurs et s'adresse à eux comme tels, dans tous les domaines de la vie (Wendy Brown, 2004, 88) ${ }^{6}$. Dans le nouveau prudentialisme, le sens moral (et notamment l'attitude prudentielle) serait désormais réduit à une affaire de délibération rationnelle sur les coûts, les bénéfices et les conséquences de telle ou telle action. Par ailleurs, adopter une attitude prudentielle ne consisterait plus tant à s'assujettir aux contenus normatifs de modèles imposés ou proposés par l'extérieur, mais bien à mobiliser ses capacités propres afin de se prendre en charge comme acteur - tout en acceptant un contrôle externe à même d'homologuer le bien fondé du comportement entrepris.

La nouvelle pénologie, plutôt située au niveau du traitement du crime, renvoie elle à une construction de gouvernement théorisée par Feeley et Simon (1992), « qui conduit peu à peu à l'abandon des fins sociales substantielles de la pénalité (normalisation, punition) au profit de fins managériales, et qui encourage ainsi un continuum gardien, à savoir un ensemble de ressources à allouer en fonction du degré de contrôle requis par le profil de risque des individus pénalisés, mais aussi en fonction de leur coût » (Brion, 2001). Par l'abandon des fins sociales au profit d'une simple gestion de risques, on peut inclure l'abandon d'objectifs comme l'amendement, la correction, la réinsertion mais aussi, d'une certaine façon, la punition. Dans ses travaux sur le champ médico-psychologique, Castel (1981) relevait déjà, dix ans plus tôt, un constat proche de celui de Feeley et Simon. Il montrait en effet comment la psychiatrie sociale et la psychanalyse avaient progressivement baissé pavillon face à une nouvelle formule de gestion du social organisé autour d'un pôle centralisé de prévention des risques et d'un pôle convivial de prise en charge des fragilités.

Sur quelle base Feeley et Simon distinguent-ils cette nouvelle pénologie de ce qu'ils appellent eux-mêmes une vieille pénologie ? En s'appuyant sur le fait que cette dernière serait basée sur la recherche des causes sociales du crime et le traitement correctif des infracteurs. Elle serait donc motivée par ses finalités sociales (soit des finalités toujours aléatoires, notamment dans la maîtrise que le système pénal peut en avoir). Or, dans le 
cas de la nouvelle pénologie, les évaluations porteront davantage sur l'effectivité des procédures mises en place que sur les résultats qu'elles peuvent produire (Dean, 1999).

Ce jeu de définitions et d'oppositions ainsi formulé, il est maintenant possible de décrire le processus dual qui sous-tend le déplacement et la reconfiguration morale de ces deux régimes de gouvernement au sein desquels l'outil risque occupe une place centrale

\title{
Un premier usage du risque dans le gouvernement du crime : donner à l'imposition d'un ordre moral l'apparence d'un simple agencement technique
}

\author{
Nouveau prudentialisme. Un code d'accès technique plus qu'une compétence \\ sociale
}

L'atrophie des liens sociaux corrélée à l'éventail toujours plus inépuisable d'environnements institutionnels ${ }^{7}$ a amené le sujet contemporain à supporter de plus en plus mal toute forme de contact en dehors de ces environnements (Lianos, 2001). Si l'adhésion à cette toile institutionnelle requiert des compétences plus techniques que sociales, elle n'en devient pas moins pour l'individu une condition préalable à sa participation "sociale ». Le déviant est dès lors celui qui cherche à échapper à ces environnements, qui n'adhère pas « au contrôle neutre et collaboratif de cette toile; un contrôle dont le pouvoir se trouve dans le déroulement programmé des processus qui 'traitent' l'individu en enchâssant ses choix dans un contexte de coordination et d'options prédéterminées qui l'écrase » (ibid., 18). Si se protéger de l'incertitude exige une adhésion et une conformité aux procédures pré-établies de la toile, cela suppose aussi une capacité d'indépendance, à savoir une capacité à mener une existence de plus en plus isolée mais qui s'appuie sur une collaboration sans faille avec les environnements institutionnels au « service » de l'individu. Nous reviendrons sur cette indépendance dans notre exploration d'un deuxième usage du risque.

Autant les individus doivent assurer de manière fonctionnelle leur admission dans des environnements pré-régulés, autant ces derniers doivent, à leur tour, se donner les garanties nécessaires en vue de pouvoir vérifier la fonctionnalité de leurs usagers. Lianos (2003) montre ainsi qu'un distributeur de billet ne doit pas savoir si l'usager d'une carte bancaire est un client légitime, pas plus qu'il ne doit se demander s'il peut davantage lui faire confiance qu'hier (l'honnêteté comme la déviance ne font plus l'objet d'une négociation sociale). Le distributeur est conçu pour seulement s'assurer que ce dernier ait les bons chiffres. Tant que cette coïncidence est assurée, la transaction est autorisée. De la même façon, la présence de stèles dans un supermarché permet seulement de s'assurer que les clients paient leurs courses, et non pas de savoir s'ils adhèrent moralement au fait de devoir payer leur nourriture. Le risque qui est géré porte sur les fraudes et non sur l'avis que le consommateur pourrait avoir à l'égard de comportements frauduleux (Lianos, 2003, 441). De même qu'il est demandé aux usagers de privilégier des compétences sociotechniques sur des valeurs socioculturelles et éthiques, il sera attendu donc des environnements institutionnels (autoroutes, logiciels, supermarchés) qu'ils favorisent des codes d'accès pré-établis sur toute forme de négociation, synonyme de divergence et de résistance potentielles. Derrière les précautions, en apparence purement techniques, que mobilisent de tels environnements institutionnels, certains n'hésiteront pas à voir un ordre moral davantage préoccupé de justice sociale. Et ce, en arguant du fait 
que la «science» du risque, du moins quand elle est appliquée au gouvernement du crime, pourrait ici et là laisser sous-entendre un refus d'objectiver la délinquance à travers des catégories sociologiques classiques (classe, genre, culture, âge, religion, habitat) et ainsi prétendre rendre possible un environnement social non stratifié. Or, si des éléments semblent bien confirmer que de telles dispositions sociotechniques reflètent d'abord des positions hautement politico-morales, ils paraissent plutôt témoigner d'une " répression égalitaire » (Lianos, 2003, 440):

«Vus de l'extérieur, nombre de nouveaux dispositifs techniques paraissent orientés équitablement envers tous les usagers. Apparemment, une nouvelle égalité émerge au-dessus de toute mise en question puisqu'elle est non seulement appliquée mais garantie par la technicité même du dispositif. De ce point de vue, la machine rend pour la première fois possible un environnement social non stratifié même si cette évolution s'effectue sur un seul critère, à savoir la possibilité de faire confiance par rapport à une priorité normative précise. Pourtant le contenu de cette égalité prête à discussion. Car ce que le dispositif distribue équitablement, ce n'est pas l'égalité des usagers par rapport à la norme mais par rapport à l'infraction ; ils deviennent tous suspects d'infraction et non plus sujets présumés honnêtes. "

On peut par ailleurs douter que tous soient suspects de la même façon puisque, pour ne prendre que le cas des caméras de surveillances, il y a fort à parier que tant au niveau des choix des lieux protégés qu'à celui des lieux réellement observés à travers l'écran, le dispositif technique dans son ensemble ne fasse que reconduire des dynamiques discriminatoires plus anciennes.

\section{Nouvelle pénologie. Le primat de l'intégration systémique sur l'intégration sociale}

21 Dans un cadre où sont de plus en plus utilisées des techniques probabilistes pour calculer et cartographier la distribution des groupes et des conduites à risque de façon à en minimiser l'impact (Rose, 2000), le temps n'est peut-être pas si loin où le retour en prison, autrefois symptomatique des échecs du système, sera un signe de la "réussite » et de l'efficacité des dispositifs de contrôle (Chantraine, 2004b, 12). Prenons un exemple. Dans un Document officiel du Canada ${ }^{8}$, on retrouve maintenant définis comme suit les succès et les échecs de libération de détenus : «Par libération réussie, nous entendons soit une libération menée à terme - le délinquant demeurant dans la collectivité, sous surveillance, jusqu'à l'expiration de sa période de semi-liberté ou jusqu'à la fin de sa peine ; soit une révocation de la libération pour manquement aux conditions. Ce deuxième type de révocation est défini comme un succès parce qu'il aura permis de diminuer le risque que le délinquant présente pour la collectivité. Est par contre définie comme échec de la libération, toute révocation qui fait suite à la commission d'une nouvelle infraction du délinquant (récidive) » $(1998,8$, nous soulignons). Une révocation pour manquement aux conditions n'est ainsi plus lue comme un échec de réinsertion mais bien comme un succès de défense sociale (avec en toile de fond, l'idée que sans cette révocation décidée à temps, « cela aurait pu être bien pire !»).

Ceci dit, parler d'une gestion purement technique des populations criminalisées est néanmoins exagéré, y compris pour les groupes à risques réputés intraitables. Même dans les circuits d'exclusion, les processus de contrôle ne se limitent pas à enfermer. Sur base de risques statiques (nombre et type de délits, âge, etc.), ils visent aussi à régulièrement produire des savoirs à même d'évaluer qui peut et qui ne peut pas réintégrer les circuits d'inclusion (Ericson, Haggerty, 1997, 41 ; Rose, 2000, 333). 


\title{
Un second usage du risque dans le gouvernement du crime : remplacer un engagement moral collectif par l'édification d'un souci de l'entreprenariat de soi
}

\author{
Nouveau prudentialisme. Transformation du monde social en champ de mines \\ potentiel
}

Pour comprendre la dimension éminemment moralisante du nouveau prudentialisme, on pointera par exemple un des usages croissants de la cartographie criminelle (Dupont, Ratcliffe, 2000, 240). Celle-ci consiste à présenter voire à prédire actuariellement la distribution des risques criminogènes dans un territoire donné. Or, outre son utilisation par les forces de l'ordre, celle-ci a progressivement été utilisée en Amérique du Nord comme méthode d'information de la population sur l'évolution de la criminalité dans leurs quartiers (notamment sur celle concernant les abuseurs sexuels). N'importe quel internaute peut désormais faire apparaitre à l'écran sa propre carte de distribution des crimes, selon les critères spécifiques qu'il aura définis préalablement. En bon homo prudens, il pourrait donc en théorie identifier quel quartier ou même quelle rue éviter. L'orientation gouvernementale de telles conduites (ne pas se balader ou s'installer dans tel endroit) est moralisante au sens où il devient «non défendable » de se promener dans un lieu à propos duquel on avait pourtant toutes les informations disponibles pour déceler qu'il ne fallait pas s'y rendre.

\section{Nouvelle pénologie. Injonction à la responsabilisation et dégradation des protections}

Un processus de responsabilisation apparaît également dans la gestion - ou plutôt l'autogestion - de sanctions pénales :

«Plutôt que de supposer que tout individu adulte est 'naturellement' capable d'une action responsable, autogérée et morale, les régimes pénaux contemporains traitent cela comme un problème auquel il faut remédier par des procédures qui cherchent activement à subjectiver et à responsabiliser les individus. Pour aider les individus à rester, redevenir ou devenir sujets, on usait auparavant d'une correction à finalité spirituelle ou morale; or, les procédures carcérales actuelles sont d'abord soucieuses d'enseigner la prudence, l'autogestion, l'intégration de ce que sont des 'bons intérêts' pour la personne ou encore l'apprentissage des bonnes prises de décision. (Garland, 1997, 191, notre traduction)»

Les détenus qui se montrent "responsables " sont alors récompensés par un Plan planning sentence qui leur permet de prendre encore davantage part à l'organisation et au déroulement de leur propre punition. Il leur est en effet permis «de choisir leurs options préférées au sein d'un éventail disponible d'activités développées dans la prison, et même parfois de choisir la prison dans laquelle ils subiront leur fin de sentence " (Garland, 1997, 191). En somme, les détenus apprennent à se gouverner eux-mêmes tout en permettant à l'institution d'insister sur l'importance de les désinfantiliser, de leur montrer un certain respect (Garland, 1997, 192). Pour reprendre Martucelli $(2004,487)$, ce mouvement des injonctions à la participation aura été, comme souvent dans la modernité, ambivalent : la reconnaissance des marges d'action de ces individus ainsi que les exigences qui leur sont demandées pour qu'ils affirment leur indépendance (au sens de leur capacité à ne dépendre de personne) se sont certes accrues mais elles l'ont fait en 
même temps que s'affaiblissaient les protections, les droits ou les supports divers qui leur auraient permis d'apprécier cette reconnaissance et de faire face à cette indépendance.

En somme, qu'il s'agisse du nouveau prudentialisme ou de la nouvelle pénologie, utiliser la catégorie du risque dans le gouvernement du crime peut donc conduire à ré-affilier les infracteurs à une communauté morale présentée comme vertueuse ; leurs problématiques diverses étant alors reformulées en problèmes d'autonomie, de responsabilité de leurs propres biographies, de reconstruction éthique (Rose, 2000). Le processus de responsabilisation qui opère ici ne se fonde pas (prioritairement) sur les notions de cause ou de faute (Dodier, 1995), il se situe plutôt sur un pôle "motivationnel »; un pôle qui se base sur des valeurs psychologiques individuelles: l'initiative personnelle, l'implication individuelle, la créativité, etc. (Digneffe et al., 2002, 123). Ce processus de responsabilisation est toujours en devenir et parce qu'il n'est jamais achevé, il «produit de l'incertitude et accentue l'inquiétude (on ne sait jamais si on s'est vraiment comporté en responsable). Dès lors, ses contours s'avèrent extrêmement flous et ses ressorts particulièrement ambigus » (ibid.).

\section{Conclusion}

Concluons. L'usage du risque tel que nous l'avons présenté dans ces deux régimes de gouvernement nous paraît riche d'enseignement à plus d'un titre. Riche parce qu'il met en évidence les rapports complexes qu'entretiennent entre eux l'outil risque et les injonctions de responsabilisation et de participation. Il ne s'agit donc pas d'une simple technicisation du gouvernement du crime qui verrait par exemple peu à peu l'outil du risque supplanter des catégories comme la responsabilité et la culpabilité. Riche ensuite, parce que cet usage spécifique du risque apparaît comme le symptôme de mutations plus globales qui touchent le fonctionnement du champ de la pénalité, et notamment l'idée que protéger les victimes potentielles est aujourd'hui plus défendable que tenter de réhabiliter des délinquants après tout eux aussi... potentiels. Riche enfin, parce que la présence de l'outil risque dans le gouvernement contemporain du crime (rappelons ici que les régimes décrits de manière idéale-typique sont surtout palpables dans les pays anglo-saxons) est peut-être un indice parmi d'autres de l'amitié récente que se portent deux frères ennemis : le néolibéralisme et le néoconservatisme (O'Malley, 1999). Si l'étude des interactions entre eux reste sans aucun doute à faire, il est clair qu'au-delà de leurs différents, au niveau de leurs positions morales par exemple, il serait intéressant de travailler «sur la façon dont, étant donnée la teneur hautement moralisatrice du programme et du ton des néoconservateurs, la rationalité néolibérale 'amorale' est devenue partie prenante de l'arsenal tactique et stratégique employé pour permettre au programme néoconservateur pour gagner du terrain» (Brown, 2004, 93). Si cette hypothèse forte devait s'avérer fondée, elle déboucherait sur la reconfiguration morale des politiques criminelles et plus largement des politiques sociales élaborées dans nombre de pays occidentaux. Elle devrait ainsi faire le constat que nous assistons tout au plus au remplacement et à la complexification des processus de domination actuels. Évoquer ce qui ressemble par certains aspects à la poussée d'un ordre moral conservateur ne devra cependant pas nous dispenser, par la suite, d'éventuellement identifier des contreprocessus qui témoigneraient par exemple de la réintégration de l'usage du risque au sein de la «vieille " pénologie correctionnaliste. Gardons-nous, dès lors, de tomber dans le même piège que celui que nous dénoncions au début de cet article, à savoir succomber 
aux sirènes de la conviction et de la dénonciation rapide au détriment de celles de l'analyse. Le va-et-vient analytique que nous évoquions ci-dessus entre particularisme et montée en généralité ne doit pas substituer une approche totalisante à une autre. Il ne faudrait donc pas que la dénonciation de la pensée néo-libérale et néo-conservatrice devienne à son tour une doxa paresseuse et sans finesse qui ferait de cette "pensée » l'explication des changements de tout ordre (Franssen, 2002).

\section{BIBLIOGRAPHIE}

BAUMAN Z., 2002, Modernité et Holocauste, Paris, La Fabrique.

BECK U., 2001 (1986), La société du risque. Sur la voie d'une autre modernité, Paris, Aubier.

BRION F., 2001, Réflexions sur les fonctions et la nature de la libération conditionnelle, Revue de Droit Pénal et de Criminologie, 4-5, 409-433.

BRION F., 2003, Du souci de soi comme impératif catégorique, in Interfaces entre santé mentale \&... Actes du colloque organisé par la Ligue Bruxelloise Francophone pour la Santé mentale et la Commission Communautaire Française les 11 et 12 octobre 2001, Bruxelles.

BROWN W., 2004, Néolibéralisme et fin de la démocratie, Vacarme, 29, 86-93.

CASTEL R., 1981, La gestion des risques : de l'anti-psychiatrie à l'après-psychanalyse, Paris, Minuit.

CHANTRAINE G., 2004a, Par-delà les murs, Expériences et trajectoires en maison d'arrêt, Paris, PUF.

CHANTRAINE G., 2004b, Prison, risque, contrôle. Mutations de l'emprise carcérale, Ecorev,

Enfermement de la misère, misère de l'enfermement, $\mathrm{n}^{\circ} 15,9-13$.

CHANTRAINE G., 2004c, « Compte-rendu de lecture de "Christie, 2003, L'industrie de la punition, prison et politique pénale en Occident, Paris, Autrement" ", Revue française de sociologie, vol. 45, 2, pp. 396-398.

CHRISTIE N., 2003, L'industrie de la punition, Paris, Autrement.

CRAWFORD A., 2001, Vers une reconfiguration des pouvoirs ? Le niveau local et les perspectives de la gouvernance, Déviance et Société, 25, 3-32.

DEAN M., 1999, Governmentality. Power and Rule in Modern Society, London, Sage.

DIGNEFFE F., NACHI M., PERILLEUX T., 2002, Des contrôles sans fin(s), ou le passage de la vérification à l'autocontrôle permanent, Recherches sociologiques, 33, 1, 109-126.

DODIER N., 1995, Les hommes et les machines : la conscience collective dans les sciences technicisées, Paris, Métailié.

DUPONT B., RATCLIFFE J., 2000, Juste quelques punaises sur une carte ? Quelques considérations critiques sur la cartographie criminelle, Les Cahiers de la sécurité intérieure, 41, 3, 229-244.

DURKHEIM E., 1986 (1893), De la division du travail social, Paris, PUF.

DURKHEIM E., 1988 (1894), Les règles de la méthode sociologique, Paris, Flammarion.

DURKHEIM E., 1992 (1925), L'éducation morale, Paris, PUF. 
ELIAS N., 1975 (1939), La dynamique de l’Occident, Paris, Calmann-Lévy.

ERICSON R.V., HAGGERTY K.D., 1997, Policing the Risk Society, Toronto, University of Toronto Press.

FEELEY M., SIMON J., 1992, The New Penology : Notes on the Emerging Strategy of Corrections and its Implications, Criminology, 30, 4, 449-474.

FOUCAULT, 1980, Résumé du cours « Du gouvernement des vivants », dans M. Foucault (Ed.), Dits et écrits, II, 1976-1988, Paris, Gallimard, Quarto, 944-948.

FOUCAULT M., 1994 (1978), La gouvernementalité, in Foucault M., Dits et écrits III, Paris, Gallimard, 635-657.

FOUCAULT M., 2004, Naissance de la biopolitique. Cours au collège de France 1978-1979, édition sous la direction de F. Ewald, A. Fontana et M. Senellart, Hautes Etudes / Gallimard / Seuil.

FRANSSEN A., A., 2002, La nouvelle fabrique du sujet, Thèse de doctorat, Université Catholique de Louvain.

GARLAND D., 1990, Punishment and Modern Society, Chicago, University of Chicago Press.

GARLAND D., 1997, Governmentality and the problem of crime : Foucault, criminology, sociology, Theoretical Criminology, 1, 2, 173-214.

GARLAND D., 1998, Les contradictions de la « société punitive » : le cas britannique, Actes de la recherches en sciences sociales, 124, 49-67.

GORDON, C., 1991, Governmental Rationality: An Introduction, in G. Burchell, C. Gordon \& P. Miller (Eds.), The Foucault Effect: Studies in Governmentability, Chicago, University of Chicago Press, HUNT A., 2003, Risk and Moralization in Everyday Life, in Ericson R.V. et Doyle A. (Eds.), Risk and Morality, Toronto, University of Toronto Press, 165-192.

KAMINSKI D., 2002, Troubles de la pénalité et ordre managérial, Recherches Sociologiques, 33, 1, 87-107.

LEMKE T., 2001, The Birth of Bio-Politics : Michel Foucault's Lecture at the College de France on Neo-Liberal Governmentality, Economy and Society, 30, 2, 190-207.

LIANOS M., 2001, Le nouveau contrôle social, Paris, L'Harmattan.

LIANOS M., 2003, Le contrôle social après Foucault, Surveillance and Society, 1, 3, 431-448.

MARTUCELLI D., D., 2004, Figures de la domination, Revue française de sociologie, 45, 3, 473-497.

MARY, PH., 2001, Pénalité et gestion des risques : vers une justice « actuarielle » en Europe, Déviance et Société, 25, 1, 33-51.

MEAD G.H., 1918, The Psychology of Punitive Justice, American Journal of Sociology 23, 577-602.

O’MALLEY P., 1992, Risk, power and crime prevention, Economy and Society, 21, 252-275.

O’MALLEY P., 1999, Volatile and contradictory punishment, Theoretical Criminology, 3, 2, 175-196.

ROSE N., 2000, Government and Control, British Journal of Criminology, 40, 321-339.

SENELLART M., 2004, Situation des cours, in M. Foucault (Ed.), Sécurité, territoire, population. Cours au Collège de France, 1977-1978, Paris, Gallimard/Seuil, Hautes Études, 379-412.

SOLLICITEUR GÉNÉRAL DU CANADA, 1998, Pour une société juste, paisible et sûre: la Loi sur le système correctionnel et la mise en liberté sous condition cinq ans plus tard : rapport des consultations, 1998, $127 \mathrm{p}$. 


\section{NOTES}

1. Pour une large introduction, voir Dean, 1999.

2. Cette normativité est d'autant plus invisibilisée que le risque se présente comme une donnée « scientifique ». La perspective gouvernementaliste vise, comme nous le détaillerons, à donner à voir cette normativité.

3. Ajoutons également qu'au-delà même des processus de superposition, de coexistence simultanée et d'influences réciproques entre divers régimes de gouvernement, il faut en outre rester attentif à la manière dont les acteurs manipulent différentes rationalités dans leurs pratiques quotidiennes, comment ils les adoptent, les contournent, les redéfinissent ou comment ils leur résistent au nom d'une éthique, de valeurs, d'un pragmatisme, d'une routine, d'un savoirfaire, etc. Ce décalage ne peut malheureusement pas être analysé ici.

4. Cette notion, notamment forgée par le norvégien N. Christie (2003), mais également par d'autres sociologues critiques américains, doit sans doute son apparente radicalité à "l'excès " de l'objet qu'ils tentent de comprendre: l'incroyable inflation carcérale américaine et l'avènement d'une incarcération dite «de masse » au prétendu pays de la liberté... Pour une analyse critique de l'influence de Bauman sur Christie, voir le commentaire de Chantraine, 2004c. 5. Si Brown ne fait pas de distinction entre théoriciens du néolibéralisme, elle rappelle que dans ses cours consacrés au libéralisme en 1978 et 1979, Foucault (2004) avait pris soin de distinguer la pensée ordo-libérale de celle de l'Ecole de Chicago (qui lui succèdera tout en la radicalisant).

6. Voir aussi Foucault, 2004 et, pour une synthèse précieuse, Lemke, 2001.

7. Nous empruntons ici à Lianos $(2001,16)$ sa notion d'institution, à savoir « toute structure qui centralise le comportement humain autour de sa propre existence et de ses propres projets, et dans ce sens, configure inévitablement le fragments d'action et de pensée qui lui sont consacrés, avec un impact sur la vie interne, intime et sociale des sujets de ces fragments ».

8. Pour une société juste, paisible et sûre: la Loi sur le système correctionnel et la mise en liberté sous condition cinq ans plus tard (Solliciteur général du Canada, 1998). Nous remercions P. Landreville pour nous avoir fourni cet exemple.

\section{INDEX}

Index chronologique : XXe siècle, XXIe siècle

Keywords : risk, penology, prudentialism, morality

Mots-clés : risque, crime, pénologie, prudentialisme, moralité

Index géographique : International

\section{AUTEURS}

\section{JEAN-FRANÇOIS CAUCHIE}

Jean-François Cauchie est Professeur à l'Université d'Ottawa (Canada), chercheur attaché au CICC (Montréal), au CIRCEM (Ottawa) et à l'UCL (Belgique). 


\section{GILLES CHANTRAINE}

Gilles Chantraine est chargé de recherche au CNRS - CESDIP, Guyancourt (France). 